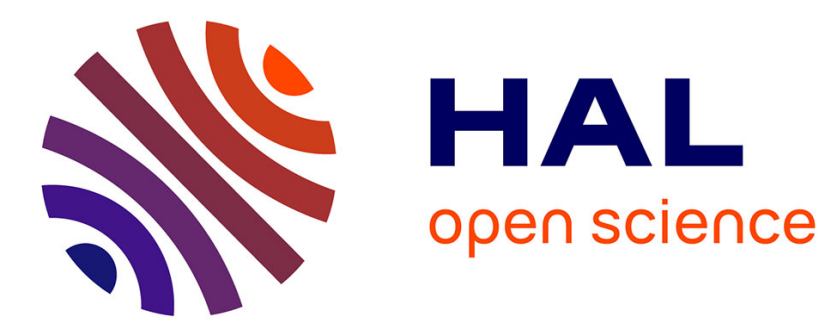

\title{
Big Content Providers Weighing on Non-Neutrality?
}

\author{
Patrick Maillé, Bruno Tuffin
}

\section{To cite this version:}

Patrick Maillé, Bruno Tuffin. Big Content Providers Weighing on Non-Neutrality?. Netnomics: Economic Research and Electronic, 2021, pp.1-20. 10.1007/s11066-021-09146-y . hal-01851791

\section{HAL Id: hal-01851791 \\ https://hal.inria.fr/hal-01851791}

Submitted on $30 \mathrm{Jul} 2018$

HAL is a multi-disciplinary open access archive for the deposit and dissemination of scientific research documents, whether they are published or not. The documents may come from teaching and research institutions in France or abroad, or from public or private research centers.
L'archive ouverte pluridisciplinaire HAL, est destinée au dépôt et à la diffusion de documents scientifiques de niveau recherche, publiés ou non, émanant des établissements d'enseignement et de recherche français ou étrangers, des laboratoires publics ou privés. 


\title{
Big Content Providers Weighing on Non-Neutrality?
}

\author{
Patrick Maillé \\ IMT Atlantique \\ Rennes, France, \\ patrick.maille@imt.fr \\ Bruno Tuffin \\ Inria Rennes Bretagne Atlantique \\ Campus Universitaire de Beaulieu, 35042 Rennes Cedex, France \\ bruno.tuffin@inria.fr
}

July 30, 2018

\begin{abstract}
There is a trend for big content providers such as Netflix and YouTube to give grades to ISPs, to incentivize those ISPs to improve at least the quality offered to their service. We design in this paper a model analyzing ISPs' optimal allocation strategies in a competitive context and in front of quality-sensitive users. We show that the optimal strategy is nonneutral, that is, it does not allocate bandwidth proportionally to the traffic share of content providers. On the other hand, we show that non-neutrality does not benefit ISPs but is surprisingly favorable to users' perceived quality.
\end{abstract}

\section{Introduction}

While the volume of data exchanged on the Internet has considerably increased in recent years [8], most of it is due to a very small number of content providers (CPs). As of 2015 in North America for example, Netflix and YouTube were accounting for more than $50 \%$ of all traffic [11]. The services those actors provide are very sensitive to the quality of the delivery of their flows to users; indeed they mostly offer high-definition video, a service that is bandwidth-consuming for the Internet Service Providers (ISPs) operating this delivery. To ensure the right service to their customers, those CPs report the quality provided by the various ISPs for their service. Google (owner of YouTube) on its web page https://www.google.com/get/videoqualityreport/ is grading ISPs, depending on your location, based on how well they are able to stream YouTube videos, providing badges "YouTube HD Verified", "standard definition" or "lower definition". Here, Google joined Netflix who was using the so-called ISP speed index (see https://ispspeedindex.netflix.com/) to measure Netflix performance on the different ISPs.

It is then natural to assume that such information will be taken into account by customers when selecting an ISP to subscribe to; the choice would be based on the qualities advertised for their preferred services. In that sense, displaying the quality information can put some relevant competitive pressure on ISPs to upgrade their network; but it may also act as an incentive for ISPs to differentiate services and favor such big providers in order to receive the best grades and attract more customers, at the expense of small providers. Such a differentiated treatment among flows is directly linked to the network neutrality debate $[3,5,7,12]$, a debate that has been active for about twenty years, with regulations passed (and sometimes undone, as in 2017 in the US) worldwide $[1,6,2]$. The debate was initiated by ISPs demanding some financial compensation from heavy resource-consuming CPs to cover their network upgrading and maintenance costs; for lack of which ISPs could block or throttle their traffic. It led to strong reactions from CPs and 
user associations, afraid of the potential impact on innovation and on the successful open network model. The pressure was then from ISPs on CPs; the threat considered in this paper is somewhat reversed: ISPs would tend to favor the biggest CPs, without any financial compensation, to attract more subscribers.

We propose in this paper a model to analyze the decisions of ISPs with regard to service differentiation of $\mathrm{CP}$ flows, in a context of two competing ISPs. Among the key components in such a game among ISPs, will be the user preferences in terms of their interests for the different CPs; we assume a distribution over the subscriber population. Based on that distribution and on its competitor's choice, each ISP has to determine the amount of capacity to devote to each CP. We therefore have a Stackelberg game [10], with

1. in a first stage, ISPs choose (in a competitive manner) the amount of their capacity to assign to each $\mathrm{CP}$;

2. in a second stage, given the ISP decisions and their own preferences, users choose what ISP to subscribe to, with congestion effects also taking place (an over-congested ISP becomes less attractive).

As leaders in the game, ISPs are assumed to be able to anticipate user reactions to their choices. We therefore analyze the game using the backward induction method.

Our goal is to investigate the best strategy of competitive ISPs in terms of capacity assignment to user-attractive CPs; should there be a differentiated treatment? What is the impact with respect to a "neutral" sharing where the capacity used for a CP is proportional to its total traffic?

We have been able to characterize user equilibria as a reaction to ISPs' decisions (the above second level of the game). Using this result, the (first level) ISP game is solved and compared with the neutral situation when ISPs allocate capacity to CPs in proportion to their traffic. Our results show that in this competitive context between ISPs, the optimal capacity allocation is non-neutral and symmetric, that is, the same proportion is allocated to each CP. The market shares of ISPs are the same for both situations, meaning that ISPs would not need to fight against neutrality. On the other hand, non-neutrality leads to different qualities at CPs but proved better average perceived quality by users; so it should surprisingly even be preferred by regulators.

The remainder of this paper is organized as follows. Section 2 presents our model and the notations. In Section 3 we analyze the second stage of the game, that is the equilibrium among users when ISP choices are fixed. Section 4 then focuses on the first game level, that is, the game played among ISPs choosing how to treat CPs to attract more users. Section 5 compares the outcomes in the Nash equilibrium and in the neutral situation (when each ISP gives to each CP a proportion capacity equal to its proportion of traffic).

Finally, Section 6 concludes and presents the most interesting extensions of the model. For a better readability of the results, most of the proofs are deferred to appendices.

This paper is an extension and a refinement of our workshop paper [9]: in [9], users also had heterogeneous preferences among CPs, but all had the same usage pattern; we consider here a more realistic model where preferences are represented by different traffic patterns. It required a complete rewriting of the proofs and modified the numerical results.

\section{Model}

This section presents our modeling choices for ISP decisions, qualities for different CPs, and user preferences. Throughout this paper, we consider two CPs, and two competing ISPs. No competition is assumed among CPs; they can either be treated as complements, or we assume that user behavior with respect to them is fixed, because of their habits (or their existing subscriptions, for paid services like Netflix). Hence the overall demand for each CP is a constant, and we focus on the ISPs possibly favoring one $\mathrm{CP}$ over the other and the consequences in terms of user subscriptions to ISPs.

ISPs will be labeled by $A$ and $B$, and CPs by 1 and 2 . We denote by $C_{i}(i=A, B)$ the total capacity of ISP $i$. 


\subsection{ISP decisions and CP-ISP qualities}

The decision variable of $\operatorname{ISP} i(i=A, B)$ is the proportion $\beta_{i} \in[0,1]$ of its total capacity $C_{i}$ that it decides to devote to $\mathrm{CP} 1$ traffic; as a direct consequence a proportion $1-\beta_{i}$ is used for $\mathrm{CP} 2$ traffic. Hence, CP1 traffic for ISP $i$ subscribers benefits from a capacity $\beta_{i} C_{i}$ and $\mathrm{CP} 2$ traffic is assigned a capacity $\left(1-\beta_{i}\right) C_{i}$.

Let us denote by $T_{i, j}$ the total (at the considered time, that is classically the peak hour) throughput corresponding to $\mathrm{CP} j$ traffic to ISP $i$ subscribers $(j=1,2 ; i=A, B)$. We consider a simple quality measure, as the ratio between the available capacity for that flow and the throughput: mathematically, the quality $Q_{i j}$ for each CP $j$ through ISP $i$ is assumed to be such that

$$
\left\{\begin{aligned}
Q_{i 1} & :=\frac{\beta_{i} C_{i}}{T_{i 1}} \\
Q_{i 2} & :=\frac{\left(1-\beta_{i}\right) C_{i}}{T_{i 2}} .
\end{aligned}\right.
$$

Based on those qualities and on their usage, which we specify in the next subsection, users will select their preferred ISP.

\subsection{User online behavior}

We assume that users differ in their relative usage of CP1 and CP2 services, which is characterized by a type for each user, that is, a value $\theta \in[0,1]$. More specifically, we consider that a type- $\theta$ user spends a proportion $\theta$ of his online time on services provided by CP1, and a proportion $1-\theta$ of his time on services provided by $\mathrm{CP} 2$, during the peak hour.

We denote by $F$ the distribution function of $\theta$, and by $\bar{\theta}$ the expected value of $\theta$, i.e.,

$$
\bar{\theta}:=\mathbb{E}[\theta]=\int_{\theta=0}^{1} \theta \mathrm{d} F(\theta)=\int_{\theta=0}^{1}(1-F(\theta)) \mathrm{d} \theta .
$$

We will assume that this distribution is "regular", in the following sense:

Assumption A The distribution function $F$ of user types is continuous and strictly increasing over its support $[0,1]$.

\section{$2.3 \quad$ CP-ISP traffic rates}

In terms of traffic (transmission rates), CPs can differ: without loss of generality we can say that one time unit on CP2 amounts to one traffic unit, while one time unit on CP1 amounts to $\gamma$ traffic units. This can correspond to different interpretations:

- a large $\gamma$ may mean that the service from $\mathrm{CP} 1$ is more bandwidth-consuming than the other $\mathrm{CP}$ (e.g., CP1 content is high-quality video while CP2 is only audio)

- but also CP1 may reduce $\gamma$ through some optimization (e.g., improving video compression).

Let us further assume that for a user, the probability of being online (consuming content from $\mathrm{CP} 1$ or CP2) during the peak hour is a constant, independent of the user's type. Without loss of generality we will assume that probability equals 1 (otherwise a simple change of units for traffic rates can be applied).

As a result, if we denote by $\Theta_{A}$ (resp., $\Theta_{B}$ ) the set of subscribers of ISP $A$ (resp., ISP $B$ ) the traffic rate of CP1 for ISP $i$ subscribers at the peak hour is

$$
T_{i 1}=\int_{\Theta_{i}} \gamma \theta \mathrm{d} F(\theta),
$$

Similarly, the traffic rate of CP2 for ISP $i$ subscribers is

$$
T_{i 2}=\int_{\Theta_{i}}(1-\theta) \mathrm{d} F(\theta) .
$$




\section{$2.4 \quad$ User preferences}

We assume that users are sensitive to the average quality they experience, i.e., a type- $\theta$ user is sensitive to

$$
\theta Q_{i 1}+(1-\theta) Q_{i 2}
$$

with $Q_{i j}$ the quality she experiences with $\mathrm{CP} j$ if subscribing to ISP $i$, as given in (1). If user subscription prices are identical-an assumption we make in this paper-then each type- $\theta$ user is assumed to subscribe to the ISP providing the highest average quality for him.

Note that we consider ISP prices as fixed, and sufficiently low, so that all users subscribe to one and only one ISP: a no-subscription option would indeed deprive users from basic services such as e-mail, whose value is assumed above the ISP subscription prices, and subscribing to two ISPs is supposed to be too costly for the possible quality improvement it would yield.

\subsection{User equilibrium}

Consider fixed values of ISP capacities $\left(C_{A}, C_{B}\right)$, treatment of $\mathrm{CP} 1$ with regard to $\mathrm{CP} 2$ (the values $\beta_{A}$ and $\beta_{B}$ ), and prices (not considered in the model, but assumed constant and the same for both ISPs).

Then our model describes interactions among users, through congestion: more users selecting an ISP leads to more traffic on that ISP, thus poorer qualities for both CPs, making the ISP less attractive. This leads to the notion of an equilibrium among users [10, ?]: each user will have a preferred ISP that will depend on her type, and on the choices of the other users, an equilibrium being a situation from which no user wants to switch to a strictly preferred ISP.

For our model, a user equilibrium would be defined as follows.

Definition 1 For given capacity values $C_{A}, C_{B}$, capacity repartitions $\beta_{A, 1}, \beta_{B, 1}, C P 1$ rate factor $\gamma$, and user repartition $F$, a user equilibrium is a partition of the interval $[0,1]$ into two domains $\Theta_{A}$ and $\Theta_{B}$, such all users in $\Theta_{i}(i=A, B)$ prefer ISPi over the competing ISP. Mathematically,

$$
\theta \in \Theta_{i} \quad \Rightarrow \quad \theta Q_{i 1}+(1-\theta) Q_{i 2} \geq Q_{\bar{i} 1}+(1-\theta) Q_{\bar{i} 2}
$$

for $i \in\{A, B\}$ and $\bar{i}=\{A, B\} \backslash\{i\}$.

User equilibria are analyzed in Section 3.

\subsection{ISP preferences and decision variables}

Given that we assumed prices fixed, ISP revenues are proportional to their market share. Hence we consider that ISP $i$ 's utility is simply its market share, that is,

$$
m_{i}:=\int_{\Theta_{i}} \mathrm{~d} F(\theta)
$$

for $i=A, B$, where $\Theta_{i}$ denotes the set of user types preferring ISP $i$ over its competitor. Those sets are considered at a user equilibrium, and depend on the ISP capacities $C_{A}$ and $C_{B}$, and on the ISP decisions whether to favor a CP or another (namely, the variables $\beta_{A}$ and $\beta_{B}$ ).

The game among ISPs is played on those decision variables $\beta_{A}$ and $\beta_{B}$, anticipating the subsequent user choices. That game is studied in Section 4 .

\subsection{Reference case: the "neutral" situation}

Our main goal, besides determining the solution of the capacity assignment game of previous subsection, is to compare the results with the situation of a "neutral" assignment where each ISP's capacity share for CP1 equals the proportion of $\mathrm{CP} 1$ traffic in the total traffic. This is formalized below. 
Definition 2 We say that ISPi chooses a neutral behavior if $\beta_{i}=\beta^{\text {neut }}$, with

$$
\beta^{\text {neut }}:=\frac{\int_{0}^{1} \gamma \theta \mathrm{d} F(\theta)}{\int_{0}^{1} \gamma \theta \mathrm{d} F(\theta)+\int_{0}^{1}(1-\theta) \mathrm{d} F(\theta)}=\frac{\gamma \bar{\theta}}{\gamma \bar{\theta}+1-\bar{\theta}}=\frac{1}{1+\frac{1}{\gamma}(1 / \bar{\theta}-1)} .
$$

Note that $\beta^{\text {neut }}=\bar{\theta}$ if and only if $\gamma=1$, indeed a bit of algebra yields $\beta^{\text {neut }}=\bar{\theta} \Leftrightarrow 1-\bar{\theta}=\frac{1}{\gamma}(1-\bar{\theta})$, with $\bar{\theta}<1$ under Assumption A.

Such a neutral behavior could be seen as corresponding to what net neutrality proponents suggest, i.e., all types of traffic receive the same treatment. In the rest of our analysis, we will remark that this situation is unlikely to be an equilibrium in the game played by ISPs, unless $\gamma=1$ (i.e., both CPs have the same data rate toward users).

The underlying question is: are the results (in particular, ISP subscription levels) significantly different in the neutral and non-neutral cases? Should the behavior of ISPs be monitored and regulated? Should big CPs be asked to be less aggressive in advertising ISPs' performance?

\section{Analysis of user equilibria}

ISPs anticipating users' reaction to their strategy when playing their game, we first need to determine users equilibria for any set of parameters.

Let us consider fixed capacities $C_{A}>0, C_{B}>0$ and $\mathrm{CP} 1$ rate factor $\gamma$. In this section we also assume fixed decision variables $\beta_{A}, \beta_{B} \in[0,1]^{2}$, to focus on the equilibria of the game played among users selecting their ISP.

We first rule out some situations, that cannot be equilibrium outcomes.

Lemma $1 A$ situation where an ISP attracts no demand $\left(m_{i}=0\right.$ for some $\left.i \in\{A, B\}\right)$ cannot be an equilibrium. As a consequence, the qualities must satisfy one of the three conditions below:

1. $Q_{A 1}=Q_{B 1}$ and $Q_{A 2}=Q_{B 2}$,

2. $Q_{A 1}>Q_{B 1}$ and $Q_{A 2}<Q_{B 2}$,

3. $Q_{A 1}<Q_{B 1}$ and $Q_{A 2}>Q_{B 2}$.

Proof: Assume $m_{A}=0$. Then $T_{A 1}=T_{A 2}=0$, hence from (1) the qualities $Q_{A 1}$ and $Q_{A 2}$ are infinitely large. On the other hand $T_{B 1}>0$ and $T_{B 2}>0$, hence finite qualities for both CPs with ISP $B$. This means that all users would prefer to switch to ISP $A$ : the current situation is not an equilibrium. Inverting the ISP indices, we must also have $m_{B}>0$ at a user equilibrium.

The second part of the proof is straightforward: if we are not in one of the situations listed in the lemma, then one ISP is strictly preferred by all users (except possibly a mass 0 ), for example if $Q_{A 1}>Q_{B 1}$ and $Q_{A 2} \geq Q_{B 2}$ all users with $\theta<1$ strictly prefer ISP $A$, and under Assumption A we would have $m_{B}=0$, a contradiction.

Definition 3 To simplify notations, we define

$$
\begin{aligned}
C_{1} & :=\beta_{A} C_{A}+\beta_{B} C_{B} \\
C_{2} & :=\left(1-\beta_{A}\right) C_{A}+\left(1-\beta_{B}\right) C_{B} \\
\alpha_{1} & :=\frac{\beta_{A} C_{A}}{C_{1}}=\frac{\beta_{A} C_{A}}{\beta_{A} C_{A}+\beta_{B} C_{B}} \\
\alpha_{2} & :=\frac{\left(1-\beta_{A}\right) C_{A}}{C_{2}}=\frac{\left(1-\beta_{A}\right) C_{A}}{\left(1-\beta_{A}\right) C_{A}+\left(1-\beta_{B}\right) C_{B}} .
\end{aligned}
$$

In words: $\alpha_{1}$ (resp., $\alpha_{2}$ ) represents the contribution (proportion of capacity) from ISP $A$ to the overall capacity $C_{1}$ (resp., $C_{2}$ ) offered to $\mathrm{CP} 1$ (resp., CP2). The complements $1-\alpha_{1}$ (resp., $1-\alpha_{2}$ ) are of course the proportions provided by ISP $B$.

We also define the functions $G$ and $H$, which will be used in the reasoning. 
Definition 4 Let us denote by $G(x)$ (resp, $H(x)$ ) the expected demand-proportion of the total user mass-for CP1 (resp., CP2) from the mass $x$ of users least interested in CP1. Formally,

$$
\begin{aligned}
\forall x \in[0,1], \quad G(x) & :=\int_{0}^{x} \theta \mathrm{d} F(\theta) \\
H(x) & :=\int_{0}^{x}(1-\theta) \mathrm{d} F(\theta) .
\end{aligned}
$$

Under Assumption A, $G$ is a continuous and strictly increasing bijection from $[0,1]$ to $[0, \bar{\theta}]$, and $H$ is a continuous and strictly increasing bijection from $[0,1]$ to $[0,1-\bar{\theta}]$.

\subsection{User equilibria: analytical characterization}

Using the previous definitions, we first analyze the conditions to fall within Case 1 of Lemma 1. Recall that proofs can be found in appendix.

Lemma $2 A$ user equilibrium with $Q_{A 1}=Q_{B 1}$ and $Q_{A 2}=Q_{B 2}$ exists if and only if

$$
1-F\left(G^{-1}\left(\left(1-\alpha_{1}\right) \bar{\theta}\right)\right) \leq \alpha_{1} \bar{\theta}+\alpha_{2}(1-\bar{\theta}) \leq F\left(G^{-1}\left(\alpha_{1} \bar{\theta}\right)\right)
$$

in which case we have

$$
\left\{\begin{array}{l}
T_{A 1}=\gamma \alpha_{1} \bar{\theta} \\
T_{B 1}=\gamma\left(1-\alpha_{1}\right) \bar{\theta} \\
T_{A 2}=\alpha_{2}(1-\bar{\theta}) \\
T_{B 2}=\left(1-\alpha_{2}\right)(1-\bar{\theta}) .
\end{array}\right.
$$

The market shares $m_{A}$ and $m_{B}$ are unique, and given by

$$
\left\{\begin{array}{l}
m_{A}=\alpha_{1} \bar{\theta}+\alpha_{2}(1-\bar{\theta}) \\
m_{B}=1-\alpha_{1} \bar{\theta}-\alpha_{2}(1-\bar{\theta}) .
\end{array}\right.
$$

We now focus on the other possible types of equilibria.

Lemma 3 Under Assumption $A$, a user equilibrium with $Q_{A 1}>Q_{B 1}$ and $Q_{A 2}<Q_{B 2}$ exists if and only if $F\left(G^{-1}\left(\left(1-\alpha_{1}\right) \bar{\theta}\right)\right)<1-\alpha_{1} \bar{\theta}-\alpha_{2}(1-\bar{\theta})$. In that case, it is unique and of the form $\Theta_{A}=\left[\theta^{*}, 1\right]$ and $\Theta_{B}=\left[0, \theta^{*}\right)$, with $\theta^{*}$ the unique solution over the open interval $\left.\left(G^{-1}\left(\left(1-\alpha_{1}\right) \bar{\theta}\right), H^{-1}\left(\left(1-\alpha_{2}\right)(1-\bar{\theta})\right)\right)\right)$ of the fixed-point equation

$$
x=\frac{1}{1+\frac{1}{\gamma} \frac{\frac{\beta_{A} C_{A}}{G(1)-G(x)}-\frac{\beta_{B} C_{B}}{G(x)}}{\frac{\left(1-\beta_{B}\right) C_{B}}{H(x)}-\frac{\left(1-\beta_{A}\right) C_{A}}{H(1)-H(x)}}}
$$

which can also be written

$$
x=\frac{1}{1+\frac{C_{1}}{\gamma C_{2}} \frac{\frac{\alpha_{1}}{G(1)-G(x)}-\frac{1-\alpha_{1}}{G(x)}}{\frac{1-\alpha_{2}}{H(x)}-\frac{\alpha_{2}}{H(1)-H(x)}} .}
$$

Exchanging the roles of ISPs in Lemma 3 and following the same steps, we also get the following result.

Lemma 4 Under Assumption $A$, a user equilibrium with $Q_{A 1}<Q_{B 1}$ and $Q_{A 2}>Q_{B 2}$ exists if and only if $F\left(G^{-1}\left(\alpha_{1} \bar{\theta}\right)\right)<\alpha_{1} \bar{\theta}+\alpha_{2}(1-\bar{\theta})$. In that case, it is unique and of the form $\Theta_{A}=\left[0, \theta^{*}\right]$ and $m_{B}=\left(\theta^{*}, 1\right]$, with $\theta^{*}$ the unique solution over the open interval $\left(G^{-1}\left(\alpha_{1} \bar{\theta}\right), H^{-1}\left(\alpha_{2}(1-\bar{\theta})\right)\right)$ of the fixed-point equation

$$
x=\frac{1}{1+\frac{1}{\gamma} \frac{\frac{\beta_{B} C_{B}}{G(1)-G(x)}-\frac{\beta_{A} C_{A}}{G(x)}}{\frac{\left(1-\beta_{A}\right) C_{A}}{H(x)}-\frac{\left(1-\beta_{B}\right) C_{B}}{H(1)-H(x)}}}
$$

which can also be written

$$
x=\frac{1}{1+\frac{C_{1}}{\gamma C_{2}} \frac{\frac{1-\alpha_{1}}{G(1)-G(x)}-\frac{\alpha_{1}}{G(x)}}{\frac{\alpha_{2}}{H(x)}-\frac{1-\alpha_{2}}{H(1)-H(x)}} .}
$$


We are now ready to completely characterize the user equilibrium.

Proposition 1 Under Assumption A, for any values of the capacities $C_{A}, C_{B}$ and any ISP choices $\beta_{A}, \beta_{B} \in[0,1]^{2}$ (and hence any value of $C_{1}, C_{2}, \alpha_{1}, \alpha_{2}$ ), there is a user equilibrium, and the corresponding qualities and demands for each ISP are unique. More precisely, define the two inequalities

$$
\begin{aligned}
\alpha_{1} \bar{\theta}+\alpha_{2}(1-\bar{\theta}) & \leq F\left(G^{-1}\left(\alpha_{1} \bar{\theta}\right)\right) \\
1-\left(\alpha_{1} \bar{\theta}+\alpha_{2}(1-\bar{\theta})\right) & \leq F\left(G^{-1}\left(\left(1-\alpha_{1}\right) \bar{\theta}\right)\right) .
\end{aligned}
$$

Then,

1. if both (11) and (12) hold, then there is at least a user equilibrium, and the quantities $\left(T_{i j}\right)_{i=A, B ; j=1,2}$ as well as $m_{A}, m_{B}$ are unique, given in (9) and (10). Service qualities verify $Q_{A 1}=Q_{B 1}$ and $Q_{A 2}=Q_{B 2}$, i.e., users are indifferent between both ISPs.

2. If (11) is violated, then there is a unique (up to a measure-zero user set) user equilibrium, of the form $\left\{\begin{array}{l}\Theta_{A}=\left[0, \theta^{*}\right] \\ \Theta_{B}=\left(\theta^{*}, 1\right]\end{array}\right.$, with $\theta^{*}$ the unique solution in the interval $\left(G^{-1}\left(\alpha_{1} \bar{\theta}\right), H^{-1}\left(\alpha_{2}(1-\bar{\theta})\right)\right)$ of the fixed-point equation

$$
x=\frac{1}{1+\frac{C_{1}}{\gamma C_{2}} \frac{\frac{1-\alpha_{1}}{G(1)-G(x)}-\frac{\alpha_{1}}{G(x)}}{\frac{\alpha}{H(x)}-\frac{1-\alpha_{2}}{H(1)-H(x)}} .}
$$

Service qualities for that equilibrium verify $Q_{A 1}<Q_{B 1}$ and $Q_{A 2}>Q_{B 2}$.

3. If (12) is violated, then there is a unique (up to a measure-zero user set) user equilibrium, of the form

$$
\left\{\begin{array}{l}
\Theta_{A}=\left[\theta^{*}, 1\right] \\
\Theta_{B}=\left[0, \theta^{*}\right)
\end{array}\right.
$$

with $\theta^{*}$ the unique solution in the interval $\left(G^{-1}\left(\left(1-\alpha_{1}\right) \bar{\theta}\right), H^{-1}\left(\left(1-\alpha_{2}\right)(1-\bar{\theta})\right)\right)$ of the fixed-point equation

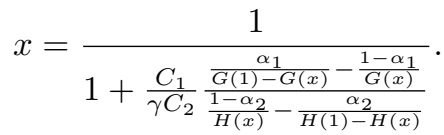

Service qualities for that equilibrium verify $Q_{A 1}>Q_{B 1}$ and $Q_{A 2}<Q_{B 2}$.

Proof: The first case stems from Lemma 2, and the two other cases from Lemmas 3 and 4.

There remains to show that those three cases cover all possibilities, i.e., that (11) and (12) cannot be jointly violated. Let us assume they are, then adding them implies

$$
1>F\left(G^{-1}\left(\alpha_{1} \bar{\theta}\right)\right)+F\left(G^{-1}\left(\left(1-\alpha_{1}\right) \bar{\theta}\right)\right) .
$$

But for any $y \in[0,1], \bar{\theta} F(y) \geq G(y)$ :

- if $y \leq \bar{\theta}$ then $\bar{\theta} F(y)-G(y)=\int_{0}^{y}(\bar{\theta}-\theta) \mathrm{d} F(\theta) \geq 0$;

- if $y>\bar{\theta}$ then $\bar{\theta} F(y)-G(y) \geq \int_{0}^{y}(\bar{\theta}-\theta) \mathrm{d} F(\theta)+\int_{y}^{1}(\bar{\theta}-\theta) \mathrm{d} F(\theta)=\int_{0}^{1}(\bar{\theta}-\theta) \mathrm{d} F(\theta)=0$.

Applying that inequality to $y=G^{-1}(x)$, we get that for all $x \in[0, \bar{\theta}], F\left(G^{-1}(x)\right) \geq x / \bar{\theta}$, so that in particular

$$
F\left(G^{-1}\left(\alpha_{1} \bar{\theta}\right)\right)+F\left(G^{-1}\left(\left(1-\alpha_{1}\right) \bar{\theta}\right)\right) \geq \alpha_{1}+1-\alpha_{1}=1,
$$

contradicting (13).

Corollary 1 For any $\left(\beta_{A}, \beta_{B}\right)$ in a vicinity of $(\beta, \beta)$ with $\beta \in(0,1)$, the user equilibrium is such that $Q_{A 1}=Q_{B 1}$ and $Q_{A 2}=Q_{B 2}$. 
In words, if ISPs choose approximately the same allocation policy (but not necessarily exactly), qualities end up being the same at both ISPs.

Proof: We first note from (6)-(7) that if $\beta_{A}=\beta_{B}=\beta$, then $\alpha_{1}=\alpha_{2}=\frac{C_{A}}{C_{A}+C_{B}}$, so that the left-hand side of (11) simply equals $\alpha_{1}=\frac{C_{A}}{C_{A}+C_{B}}$. Since we assumed that capacities $C_{A}$ and $C_{B}$ are strictly positive, we have $\alpha_{1} \in(0,1)$.

Then, mirroring the proof of Proposition 1, we can establish the strict inequality $\bar{\theta} F(y)>G(y)$ for $y \in(0,1)$. Indeed,

- if $0<y \leq \bar{\theta}$ then $\bar{\theta} F(y)-G(y)=\int_{0}^{y}(\bar{\theta}-\theta) \mathrm{d} F(\theta)>0$;

- if $1>y>\bar{\theta}$ then $\bar{\theta} F(y)-G(y)>\int_{0}^{y}(\bar{\theta}-\theta) \mathrm{d} F(\theta)+\underbrace{\int_{y}^{1}(\bar{\theta}-\theta) \mathrm{d} F(\theta)}_{<0}=\int_{0}^{1}(\bar{\theta}-\theta) \mathrm{d} F(\theta)=0$.

Taking $y=G^{-1}\left(\alpha_{1} \bar{\theta}\right)$, under Assumption A we have $y \in(0,1)$, so $F\left(G^{-1}\left(\alpha_{1} \theta\right)\right)>\alpha_{1}$, i.e., $(11)$ is satisfied with a strict inequality.

Similarly, taking $y=G^{-1}\left(\left(1-\alpha_{1}\right) \bar{\theta}\right)$ and noting that the left-hand side of (12) equals $1-\alpha_{1} \in$ $(0,1),(12)$ is satisfied with a strict inequality.

Hence by continuity, (11) and (12) remain satisfied within a vicinity of $(\beta, \beta)$, i.e., the corresponding equilibrium is such that $Q_{A 1}=Q_{B 1}$ and $Q_{A 2}=Q_{B 2}$.

\subsection{Illustration of user equilibria}

As an illustration, we will consider a specific distribution.

Assumption B User-preference parameters $\theta$ are distributed over the interval $[0,1]$ so that the cumulative distribution function is $F(x)=x^{\kappa}$ for some $\kappa>0$. In particular, $\kappa=1$ corresponds to a uniform distribution.

With such distributions, the functions and terms we have defined are easy to express:

- $\bar{\theta}=\int_{0}^{1} \theta \mathrm{d} F(\theta)=\frac{\kappa}{\kappa+1}$;

- for $x \in[0,1], G(x)=\int_{0}^{x} \theta \mathrm{d} F(\theta)=\frac{\kappa x^{\kappa+1}}{\kappa+1}$, so that the inverse for $y \in[0,1]$ is $G^{-1}(y \bar{\theta})=y^{\frac{1}{\kappa+1}}$ and $F\left(G^{-1}(y \bar{\theta})\right)=y^{\bar{\theta}}$;

- the two inequalities used to characterize the type of user equilibrium we get can be rewritten: (11) is equivalent to

$$
\alpha_{1} \bar{\theta}+\alpha_{2}(1-\bar{\theta}) \leq \alpha_{1}^{\bar{\theta}}
$$

while (12) is equivalent to

$$
1-\left(\alpha_{1} \bar{\theta}+\alpha_{2}(1-\bar{\theta})\right) \leq\left(1-\alpha_{1}\right)^{\bar{\theta}} .
$$

In the remainder of this paper, we will run numerical experiments under Assumption B. The value of the parameter $\kappa$, as well as the other parameters considered, are given in Table 1.

\begin{tabular}{cccccc}
$\kappa$ & $\gamma$ & $C_{A}$ & $C_{B}$ & $\beta_{A}$ & $\beta_{B}$ \\
\hline 2 & 2 & 10 & 5 & 0.3 & 0.7
\end{tabular}

Table 1: Parameter values considered in the numerical experiments (unless explicitly mentioned). The distribution of user types is characterized by $\kappa$, the relative traffic rate for a CP1 customer with respect to a CP2 customer is $\gamma$, and each ISP $i=A, B$ allocates a proportion $\beta_{i}$ of its capacity $C_{i}$ to $\mathrm{CP} 1$, the rest being for $\mathrm{CP} 2$ traffic.

Figures 1 to 3 display the user equilibrium situation when the parameter $\beta_{A}$ varies. Qualities for each pair (ISP,CP) are shown in Figure 1, illustrating the three possible types of user equilibrium: 


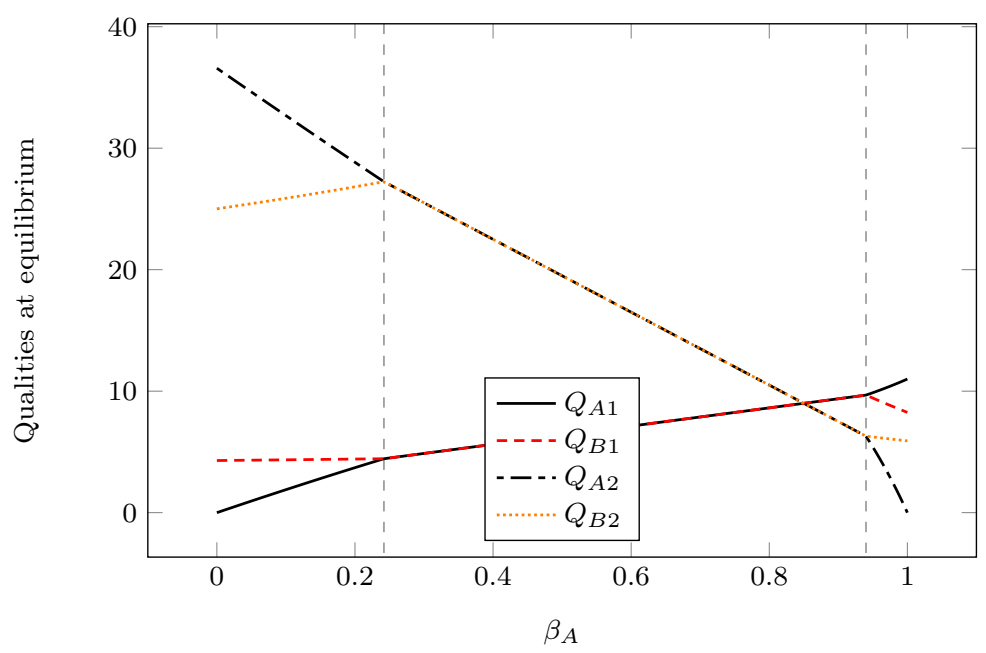

Figure 1: Qualities at equilibrium versus $\beta_{A}$. The vertical dashed lines indicate the frontiers between equilibrium zones.

- $\beta_{A}$ close to 0 means that ISP $A$ favors $\mathrm{CP} 2$ over $\mathrm{CP} 1$, which translates into quality differences: ISP $A$ performs better for CP2 and worse for $\mathrm{CP} 1$ than its competitor, hence it is chosen by users most sensitive to $\mathrm{CP} 2$ (low $\theta$ values);

- in the other extreme ( $\beta$ close to 1 ) we observe a symmetric behavior: since it devotes most of its capacity to CP1 traffic, ISP $A$ is preferred by users using mostly CP1 (high $\theta$ values), and users mostly interested in $\mathrm{CP} 2$ prefer to go to $\operatorname{ISP} B$, where the quality for $\mathrm{CP} 2$ is higher;

- between those values, both ISPs provide the same quality, for each CP, i.e., $Q_{A 1}=Q_{B 1}$ and $Q_{A 2}=Q_{B_{2}}$, therefore each user is indifferent between the ISPs. Note that for $\beta_{A}$ close to $\beta_{B}$ we are in that situation, as was established in Corollary 1. Remark also that the qualities are not generally equal among CPs, that is $Q_{A 1} \neq Q_{A 2}$ for example.

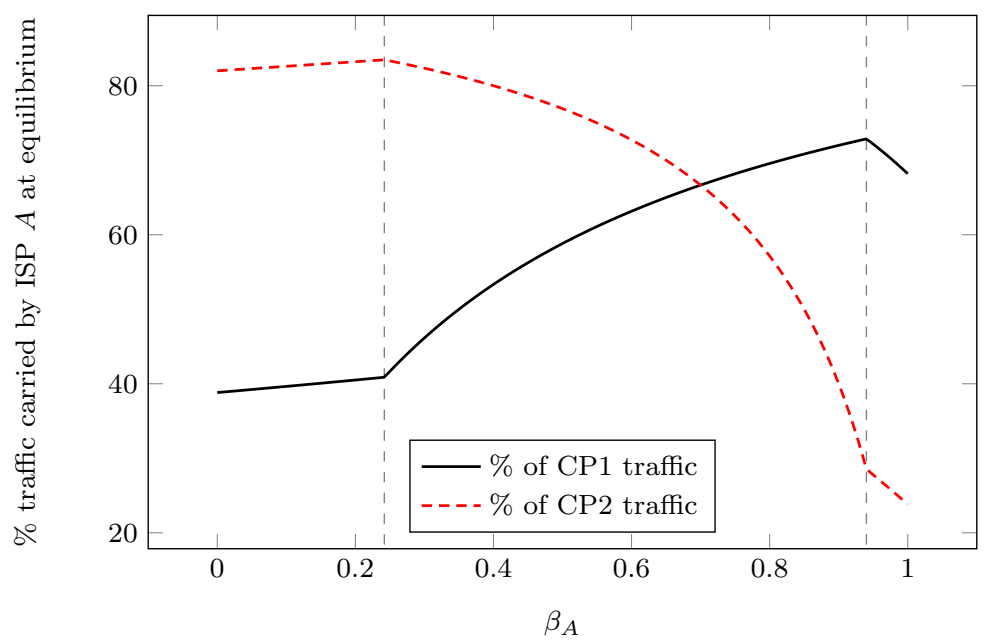

Figure 2: Proportions (percentages) of traffic from each CP carried by ISP $A$ at user equilibrium, versus $\beta_{A}$. The vertical dashed lines indicate the frontiers between equilibrium zones.

The corresponding repartition of CP1 and CP2 traffic among ISPs is shown in Figure 2, where we plot the percentage of each CP's traffic that is carried by ISP $A$ (the remaining part being taken 
by ISP $B$ ). Again the three zones are visible. In the middle zone (where both ISPs have equal qualities), the values follow the intuition: increasing $\beta_{A}$ leads to ISP $A$ carrying more CP1 traffic and less CP2 traffic.

But that does not necessarily hold in the two extreme zones. An interpretation is as follows, for example in the "high- $\beta_{A}$ " region: increasing even more $\beta_{A}$ means that the quality for CP2 significantly decreases, driving some users-and their CP1 traffic as well-away to ISP $B$. Finally,

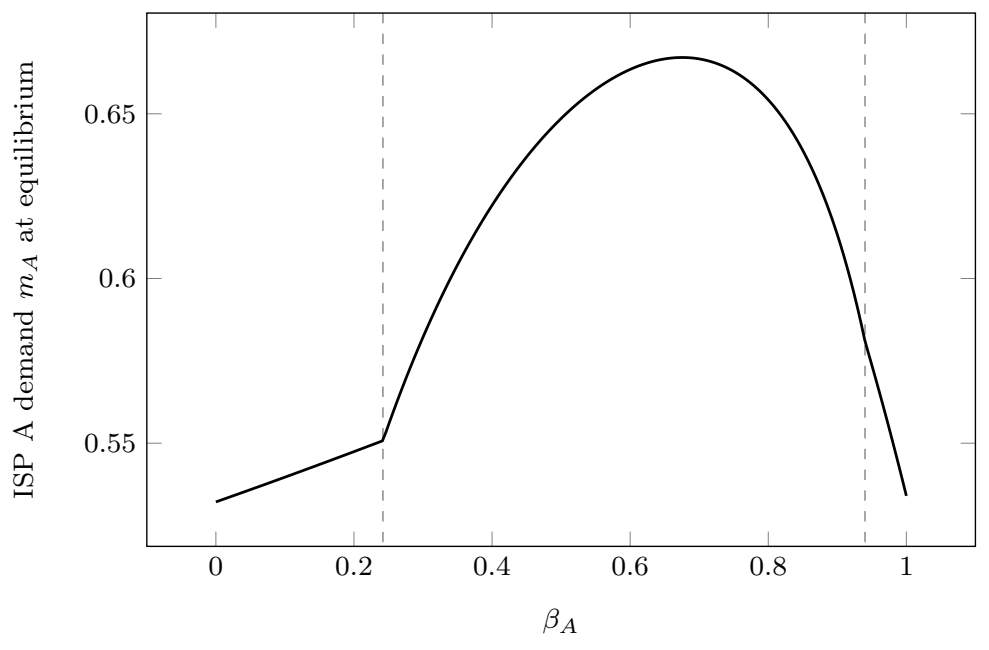

Figure 3: Demand for ISP A at user equilibrium versus $\beta_{A}$. The vertical dashed lines indicate the frontiers between equilibrium zones.

Figure 3 displays the market share of ISP $A$ at the user equilibrium, depending on $\beta_{A}$. Here the maximum is in the middle zone, i.e., when $Q_{A 1}=Q_{B 1}$ and $Q_{A 2}=Q_{B 2}$; as we will see numerically later, this is often the case, but not always.

In the rest of this paper, we will focus on ISP decisions to favor or not a CP over the other, through the share $\beta$ that they reserve for CP1 traffic. We assume ISPs make their decision in order to maximize their revenue, i.e., their market share, anticipating the subsequent user decisions characterized in Proposition 1.

\section{ISP game on CPs treatment}

In this paper, we assume that ISP subscription prices are fixed and equal (e.g., because of regulation or previous price competition among ISPs). The lever that we consider for each ISP $i(i \in\{A, B\})$ is the repartition of its capacity between CP1 (being allocated a proportion $\beta_{i}$ of ISP $i$ 's capacity $C_{i}$ ) and $\mathrm{CP} 2$ (being allocated the remaining capacity $\left(1-\beta_{i}\right) C_{i}$ ).

We study in this section the non-cooperative game played by ISPs on that capacity sharing and compare the outcome with the neutral sharing introduced in Definition 2. Recall that $\beta^{\text {neut }}=$ $\frac{1}{1+\frac{1}{\gamma}(1 / \bar{\theta}-1)}$ and that from elementary computations under Assumption $\mathrm{B}$, we have $\beta^{\text {neut }}=\frac{1}{1+\frac{1}{\kappa \gamma}}$.

We noticed previously (see Figure 3 ) that the optimal $\beta_{i}$ for an ISP $i$ can be in the interior of the interval where ISPs offer identical qualities, for each ISP. Determining analytically when it is the case is not trivial, however we can express the corresponding value.

Proposition 2 When the best-response of ISPi to ISPj's choice $\beta_{j}(j \neq i)$ is in the interior of the "equal qualities" interval, then its expression is

$$
\bar{\beta}_{i}\left(\beta_{j}\right):=\frac{1+\frac{C_{j}}{C_{i}}}{1+\sqrt{(1 / \bar{\theta}-1)\left(1 / \beta_{j}-1\right)}}-\frac{C_{j}}{C_{i}} \beta_{j} .
$$


We call that expression the pseudo-best-response of ISPi.

Proof: Let us assume that $i=A$, then the case of $i=B$ will be obtained by symmetry.

When the user equilibrium is such that $Q_{A 1}=Q_{B 1}$ and $Q_{A 2}=Q_{B 2}$, we know from (10) that the market share (hence, the utility) of ISP $A$ is $m_{A}=\alpha_{1} \bar{\theta}+\alpha_{2}(1-\bar{\theta})$, which gives, using (6) and (7),

$$
m_{A}=\frac{\bar{\theta}}{1+\frac{\beta_{B} C_{B}}{\beta_{A} C_{A}}}+\frac{1-\bar{\theta}}{1+\frac{\left(1-\beta_{B}\right) C_{B}}{\left(1-\beta_{A}\right) C_{A}}} .
$$

Differentiating with respect to $\beta_{A}$, we get

$$
\frac{\bar{\theta} \beta_{B} C_{A} C_{B}}{\left(\beta_{A} C_{A}+\beta_{B} C_{B}\right)^{2}}-\frac{(1-\bar{\theta})\left(1-\beta_{B}\right) C_{A} C_{B}}{\left(\left(1-\beta_{A}\right) C_{A}+\left(1-\beta_{B}\right) C_{B}\right)^{2}},
$$

which is strictly decreasing in $\beta_{A}$. Hence the expression in (15) is concave in $\beta_{A}$ and is maximum where its derivative is null, i.e., when

$$
\frac{\beta_{A} C_{A}+\beta_{B} C_{B}}{\sqrt{\bar{\theta} \beta_{B}}}=\frac{\left(1-\beta_{A}\right) C_{A}+\left(1-\beta_{B}\right) C_{B}}{\sqrt{(1-\bar{\theta})\left(1-\beta_{B}\right)}},
$$

or again

$\beta_{A} C_{A}\left(\frac{1}{\sqrt{\bar{\theta} \beta_{B}}}+\frac{1}{\sqrt{(1-\bar{\theta})\left(1-\beta_{B}\right)}}\right)=\frac{C_{A}+C_{B}}{\sqrt{(1-\bar{\theta})\left(1-\beta_{B}\right)}}-\beta_{B} C_{B}\left(\frac{1}{\sqrt{\bar{\theta} \beta_{B}}}-\frac{1}{\sqrt{(1-\bar{\theta})\left(1-\beta_{B}\right)}}\right)$.

Multiplying both sides of that equality by $\sqrt{\hat{\theta} \beta_{B}(1-\bar{\theta})\left(1-\beta_{B}\right)}$ gives

$$
\left(\beta_{A} C_{A}+\beta_{B} C_{B}\right)\left(\sqrt{\bar{\theta} \beta_{B}}+\sqrt{(1-\bar{\theta})\left(1-\beta_{B}\right)}\right)=\left(C_{A}+C_{B}\right) \sqrt{\bar{\theta} \beta_{B}},
$$

and finally $\beta_{A}=\frac{C_{A}+C_{B}}{C_{A}} \frac{\sqrt{\bar{\theta} \beta_{B}}}{\sqrt{\bar{\theta} \beta_{B}}+\sqrt{(1-\bar{\theta})\left(1-\beta_{B}\right)}}-\frac{C_{B}}{C_{A}} \beta_{B}$, that is, the expression in (14).

Note in particular that the expression in (14) does not depend on $\gamma$, while the "neutral" behavior of Definition 2 involves that parameter.

Again, we do not claim that (14) always provides the best-response $\beta_{i}$, since that best-response can be outside of the interior of the equal-qualities' zone. Nevertheless, analyzing the intersection of those "pseudo-best-responses" provides some interesting insight.

Proposition 3 The pseudo-best-response mapping $\left(\beta_{A}, \beta_{B}\right) \mapsto\left(\bar{\beta}_{A}\left(\beta_{B}\right), \bar{\beta}_{B}\left(\beta_{A}\right)\right)$ with $\bar{\beta}_{i}(\cdot)$ given in (14) has exactly three fixed points, that are all with symmetric values and among which only one is non-degenerate:

- $\beta_{A}=\beta_{B}=0$,

- $\beta_{A}=\beta_{B}=1$,

- $\beta_{A}=\beta_{B}=\bar{\theta}$.

Hence the only possible Nash equilibrium in the interior of the equal-quality zone is $\beta_{A}=\beta_{B}=\bar{\theta}$.

Proof: Consider an ISP $i$ and its competitor $j$. We first note that $(\bar{\theta}, \bar{\theta})$ is indeed a fixed point, by applying (14) and observing that $\bar{\beta}_{i}(\bar{\theta})=\frac{1+\frac{C_{j}}{C_{i}}}{1+(1 / \bar{\theta}-1)}-\frac{C_{j}}{C_{i}} \bar{\theta}=\bar{\theta}$. Extending by continuity the expression (14) to the values 0 and 1 give the two other fixed points.

We now prove that there cannot be any other fixed point, in two steps. 
- First, we establish that a fixed point $\left(\beta_{A}, \beta_{B}\right)$ is necessarily symmetric, i.e., such that $\beta_{A}=$ $\beta_{B}$. Indeed, a fixed point would be a solution of the system

$$
\left\{\begin{aligned}
\beta_{A} & =\frac{1+\frac{C_{B}}{C_{A}}}{1+\sqrt{(1 / \bar{\theta}-1)\left(1 / \beta_{B}-1\right)}}-\frac{C_{B}}{C_{A}} \beta_{B} \\
\frac{C_{B}}{C_{A}} \beta_{B} & =\frac{1+\frac{C_{B}}{C_{A}}}{1+\sqrt{(1 / \bar{\theta}-1)\left(1 / \beta_{A}-1\right)}}-\beta_{A} .
\end{aligned}\right.
$$

where the first equation is (14) with $i=A$, and the second is obtained from (14) applied with $i=B$, multiplying both sides by $\frac{C_{B}}{C_{A}}$. That system immediately yields $\sqrt{1 / \beta_{A}-1}=$ $\sqrt{1 / \beta_{B}-1}$, that is, $\beta_{A}=\beta_{B}$.

- Therefore, a fixed point is necessarily of the form $\left(\beta_{A}, \beta_{B}\right)=(x, x)$, with $x$ satisfying

$$
x=\frac{1+\frac{C_{B}}{C_{A}}}{1+\sqrt{(1 / \bar{\theta}-1)(1 / x-1)}}-\frac{C_{B}}{C_{A}} x .
$$

But for $0<x<\bar{\theta}$ (resp., $1>x>\bar{\theta}$ ), the right-hand side of that relation is strictly above $\frac{1+\frac{C_{B}}{C_{A}}}{1+\sqrt{(1 / \bar{\theta}-1)(1 / \bar{\theta}-1)}}-\frac{C_{B}}{C_{A}} x=\bar{\theta}\left(1+\frac{C_{B}}{C_{A}}\right)-\frac{C_{B}}{C_{A}} x>x$ (resp., strictly below $x$ ), hence $x$ cannot be a solution. The only possibilities are then the ones highlighted in the proposition.

The second claim of the proposition is straightforward, since a Nash equilibrium is a fixed point of the best-response mapping, which coincides with the pseudo-best-response mapping when in the interior of the equal-quality zone.

Those points are illustrated in Figure 4, that shows both the pseudo-best-responses from (14), and the actual best-responses computed numerically using the values of parameters introduced in Section 3.2 .

- The two functions are different, but coincide for values of the competitor's $\beta$ that are not too close to 0 or 1 ;

- the pseudo-best-response functions intersect at the three points given in Proposition 3.

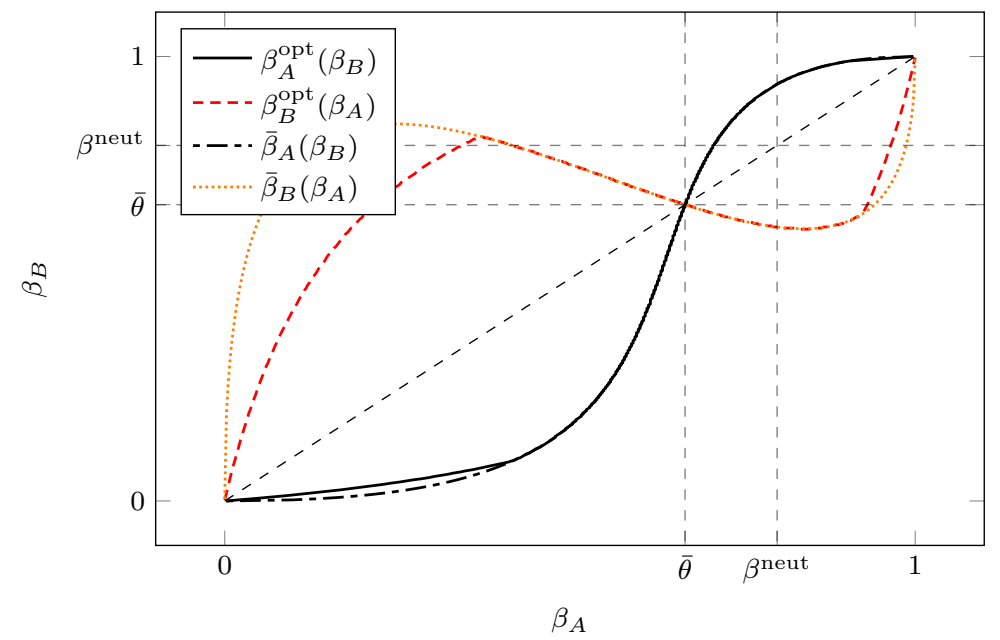

Figure 4: Best-response $\beta$ for each ISP (computed numerically), and pseudo-best-response curves from (14). The non-degenerate Nash equilibrium is $\left(\beta_{A}, \beta_{B}\right)=(\bar{\theta}, \bar{\theta})$, and we also show the neutral situation $\left(\beta_{A}, \beta_{B}\right)=\left(\beta^{\text {neut }}, \beta^{\text {neut }}\right)$.

Additionally, we observe that 
- the game on $\beta_{i}$ s has three equilibria here, that are all symmetric and correspond to the three intersection points of pseudo-best-responses;

- the extreme value points $\beta_{A}=\beta_{B}=0$ and $\beta_{A}=\beta_{B}=1$ are unstable equilibria: if one ISP slightly deviates, best-response adjustments drive decisions away from those points;

- the other Nash equilibrium $\beta_{A}=\beta_{B}=\bar{\theta}$ is stable (as can be seen from the figure: iterating best-responses, starting close to $\bar{\theta}$ converge to $\bar{\theta}$ ), and hence could be considered a reasonable prediction of the ISP interaction.

Finally, as we see next, we note that $(\bar{\theta}, \bar{\theta})$ is not always a Nash equilibrium: for extreme values of the ratio $C_{A} / C_{B}$ we found no equilibrium. Figure 5 shows the regions of $\left(C_{A}, C_{B}\right)$ for which $(\bar{\theta}, \bar{\theta})$ is an equilibrium; those regions get smaller as the parameters $\gamma$ and $\kappa$ (recall that $\bar{\theta}=\frac{\kappa}{\kappa+1}$ ) get further from 1.
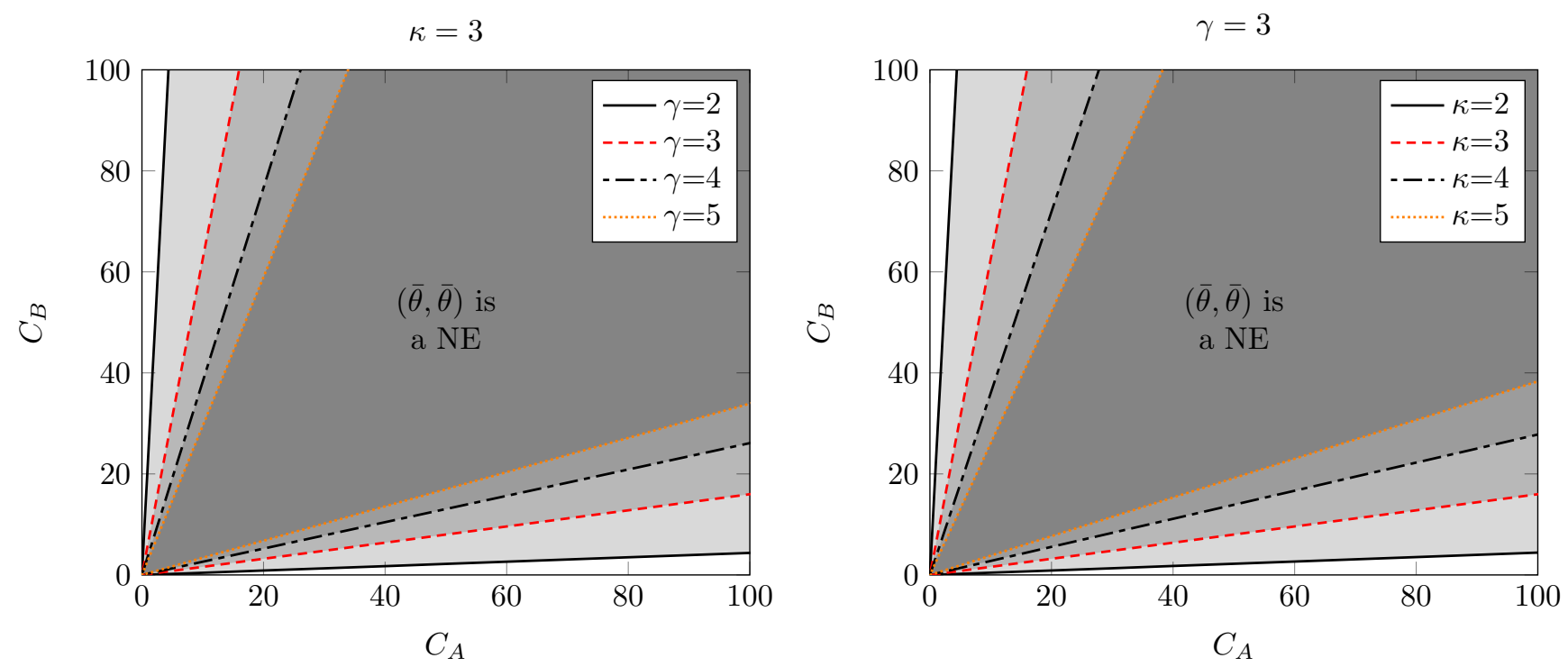

Figure 5: The $\left(C_{A}, C_{B}\right)$ region for which $(\bar{\theta}, \bar{\theta})$ is a Nash equilibrium. Outside of that region we found no equilibrium.

\section{Nash equilibrium vs neutral behavior}

Note that the only non-degenerate Nash equilibrium $(\bar{\theta}, \bar{\theta})$ found in the figure differs from a neutral behavior of Definition 2 if $\gamma \neq 1$. In this section we compare the outcomes of those two situations. Again, the figures provided in this section are obtained using the parameters given in Table 1.

\subsection{ISP market shares}

Since both the equilibrium and the neutral situations are symmetric (in the sense that $\beta_{A}=\beta_{B}$ ), then ISPs contribute to the overall capacity offered to each CP in the same proportion, i.e., from (6)-(7) we have $\alpha_{1}=\alpha_{2}=\frac{C_{A}}{C_{A}+C_{B}}$ in both cases.

As a result, (10) implies that in both cases, $m_{A}=\frac{C_{A}}{C_{A}+C_{B}}$ and $m_{B}=\frac{C_{B}}{C_{A}+C_{B}}$, meaning that ISPs are indifferent between the two cases. 


\subsection{CP treatment}

From the expression in Definition 2, we get

$$
\beta^{\text {neut }}<\bar{\theta} \quad \Leftrightarrow \quad \gamma<1 .
$$

Recall that $\gamma<1$ means that the traffic rate when connected to the CP1 service is lower than for $\mathrm{CP} 2$, which can be interpreted in two different ways:

- CP1's service is less bandwidth-consuming than CP2's by nature (e.g., text-based versus video-based)

- both providers have similar services, but CP1 has optimized its service delivery to make it less bandwidth-consuming.

When $\gamma<1$, our analysis leads to an equilibrium $(\bar{\theta}, \bar{\theta})$, i.e., each ISP favors CP1 with respect to a neutral behavior. Qualitatively, this can be justified from several points of view:

- favoring the lower-rate $\mathrm{CP}$ is consistent with policies favoring "mice" over "elephants", that maximize the number of treated flows in the Internet [4];

- the preferential treatment for rate-limited CPs can be seen as an efficient incentive for CPs to optimize their traffic (e.g., through compression), a desirable feature in the currentincreasingly congested-Internet.

\subsection{CP qualities}

We can also compute the CP qualities in both cases. Those qualities are the same on both ISPs, so we remove the dependence in the ISP to simplify notations.

- in the neutral situation, both CPs have the same quality $Q^{\text {neut }}$, that equals:

$$
Q^{\text {neut }}=\frac{C_{A}+C_{B}}{1-\bar{\theta}(1-\gamma)}
$$

- at the Nash equilibrium $(\bar{\theta}, \bar{\theta}), \mathrm{CP}$ qualities equal

$$
\begin{aligned}
& Q_{1}^{\mathrm{NE}}=\frac{C_{A}+C_{B}}{\gamma} \\
& Q_{2}^{\mathrm{NE}}=C_{A}+C_{B},
\end{aligned}
$$

i.e., $\mathrm{CP}$ qualities differ by the factor $\gamma$ : if a $\mathrm{CP}$ manages to compress its data by a given factor, the perceived quality will be improved by the same factor and the quality of the competing $\mathrm{CP}$ is unchanged, while in the neutral case the rate reduction benefits both CPs.

Those qualities are plotted in Figure 6 , where we vary $\gamma$ and $\bar{\theta}$. As the analysis shows, at the Nash equilibrium $\bar{\theta}$ has no impact on qualities, and $\gamma$ affects only CP1 quality. On the other hand, the neutral-situation qualities are between the equilibrium qualities of CP1 and CP2. All those values coincide when $\gamma=1$.

\subsection{Average perceived quality}

Finally, we can compute the quality perceived on average by users, that we can define in general as

$$
Q_{\text {Av. }}=\int_{\Theta_{A}} \theta Q_{A, 1}+(1-\theta) Q_{A, 2} \mathrm{~d} F(\theta)+\int_{\Theta_{B}} \theta Q_{B, 1}+(1-\theta) Q_{B, 2} \mathrm{~d} F(\theta) .
$$

Since in both the Nash and neutral situations the CP qualities are the same for both ISP, this simplifies into

$$
Q_{\mathrm{Av} .}=\bar{\theta} Q_{1}+(1-\bar{\theta}) Q_{2} .
$$

Hence, 


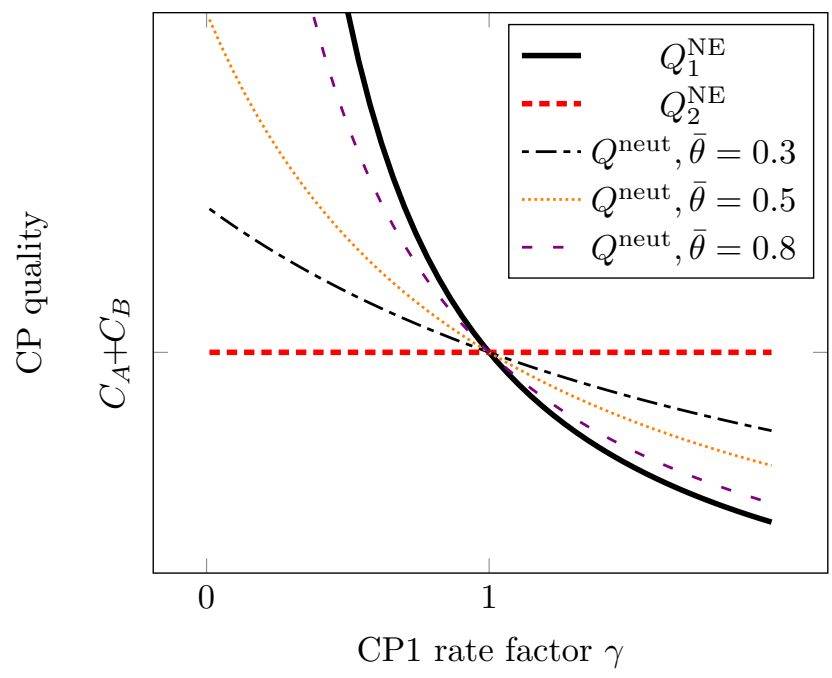

Figure 6: CP qualities at the neutral and Nash situations.

- in the neutral case, since CPs have the same quality, we simply get

$$
Q_{\mathrm{Av} .}^{\text {neut }}=Q^{\text {neut }}=\frac{C_{A}+C_{B}}{1-\bar{\theta}(1-\gamma)} ;
$$

- at the Nash equilibrium $(\bar{\theta}, \bar{\theta})$, we get

$$
Q_{\mathrm{Av} .}^{\mathrm{NE}}=\left(C_{A}+C_{B}\right)\left(1-\bar{\theta}\left(1-\frac{1}{\gamma}\right)\right) .
$$

The ratio $\frac{Q_{\mathrm{Av}}^{\mathrm{NE}}}{Q_{\mathrm{Av} \text {. }}^{\text {neut }}}$ is plotted on Figure 7 for different values of $\bar{\theta}$ and $\gamma$. We can see that the

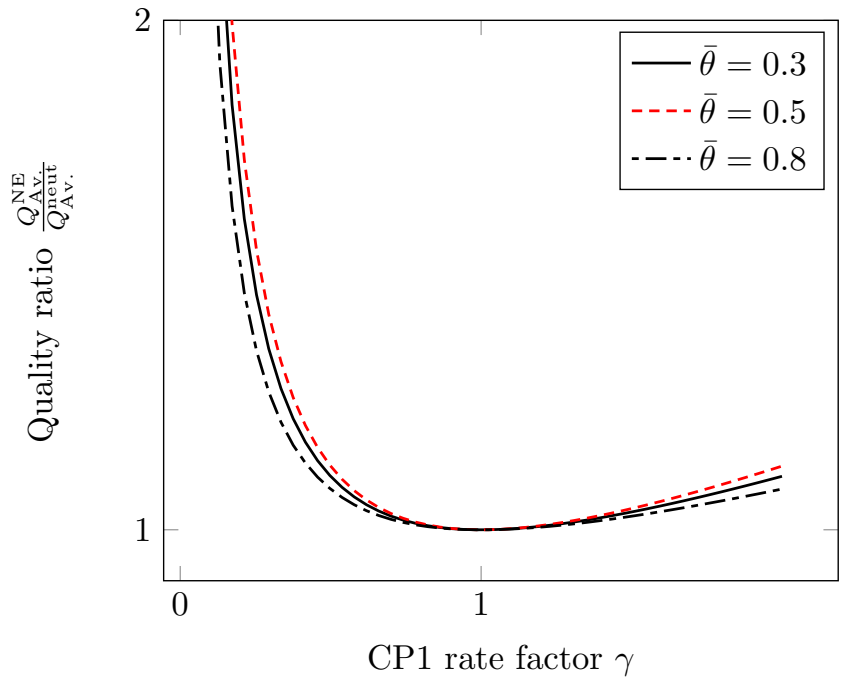

Figure 7: Relative average qualities in the equilibrium and neutral cases.

perceived quality at the Nash equilibrium is in the case always larger than that in the neutral situation. This type of result is formalized in the following proposition. 
Proposition 4 The average user perceived quality is larger when $\left(\beta_{A}, \beta_{B}\right)=(\bar{\theta}, \bar{\theta})$ than at the neutral situation.

In other terms, when $(\bar{\theta}, \bar{\theta})$ is a Nash equilibrium, a non-neutral behavior benefits to users.

Proof: Let us express the ratio of average qualities in both situations:

$$
\begin{aligned}
\frac{Q_{\mathrm{Av} .}^{\mathrm{NE}}}{Q_{\mathrm{Av} .}^{\text {neut }}} & =(1-\bar{\theta}(1-1 / \gamma)) \cdot(1-\bar{\theta}(1-\gamma)) \\
& =(1-\bar{\theta})^{2}+\bar{\theta}^{2}+\bar{\theta}(1-\bar{\theta})(\gamma+1 / \gamma) .
\end{aligned}
$$

Only the last term depends on $\gamma$, and is minimum when $\gamma=1$, in which case the ratio exactly equals 1 . Hence for all $\gamma$ and $\bar{\theta}, Q_{\mathrm{Av} \text {. }}^{\mathrm{NE}} \geq Q_{\mathrm{Av} \text {. }}^{\text {neut }}$.

\section{Conclusions}

We proposed in this paper an original model designed to analyze ISP optimal allocation strategies of capacity between content providers, in a competitive context. The main motivation of this model is based on big CPs such as Netflix and YouTube giving grades to ISPs for the quality they provide, thereby potentially incentivizing ISPs to favor their traffic. The model is analyzed as a two-level game and optimal strategies characterized.

Comparing with a neutral strategy where traffic is not differentiated, we obtain in this competitive context some surprising results: resulting market shares of ISPs are the same for both situations, but non-neutrality leads to a better average perceived quality by users. In other words, ISPs are actually indifferent between both situations while users/regulators may favor non-neutrality, the opposite of what would be a first guess. Additionally, the non-neutral strategies are likely to stimulate bandwidth usage optimization on the $\mathrm{CP}$ side, e.g., through more efficient compression. Indeed, a $\mathrm{CP}$ reducing its bandwidth usage will experience a higher quality with no impact on the competitor, while in the neutral case the improvement equally benefits to both CPs, giving no particular edge to the optimizing $\mathrm{CP}$.

This model can be extended by introducing pricing decisions by ISPs, and maybe CPs, which in turn impact users.

\section{References}

[1] Net neutrality. President Obama's plan for a free and open internet. https:// obamawhitehouse.archives.gov/node/323681. Accessed: 2018-07-24.

[2] BEREC. BEREC report on the implementation of regulation (EU) 2015/2120 and BEREC net neutrality guidelines. Technical Report BoR (17) 240, BEREC, 2017.

[3] P. Crocioni. Net neutrality in Europe: Desperately seeking a market failure. Telecommunications Policy, 35(1):1 - 11, 2011.

[4] Liang Guo and I. Matta. The war between mice and elephants. In Proceedings Ninth International Conference on Network Protocols. ICNP 2001, pages 180-188, Nov 2001.

[5] T.M. Lenard and R.J. May (Eds.). Net Neutrality or Net Neutering: Should Broadband Internet Services be Regulated. Springer, 2006.

[6] S. Lohr. Net neutrality is Trump's next target, administration says. https://www. nytimes . com/2017/03/30/technology/net-neutrality.html. Accessed: 2018-07-24. 
[7] P. Maillé, P. Reichl, and B. Tuffin. Internet governance and economics of network neutrality. In A. Hadjiantonis and B. Stiller, editors, Telecommunications Economics - Selected Results of the COST Action IS0605 EconTel, pages 108-116. Lecture Notes in Computer Science 7216, Springer Verlag, 2012.

[8] P. Maillé and B. Tuffin. Telecommunication Network Economics: From Theory to Applications. Cambridge University Press, 2014.

[9] Patrick Maillé and Bruno Tuffin. Non-Neutrality Pushed by Big Content Providers. In Congduc Pham, Jörn Altmann, and José Ángel Bañares, editors, Economics of Grids, Clouds, Systems, and Services (GECON'2017), pages 29-39, Biarritz, France, September 2017. Springer International Publishing.

[10] M. Osborne and A. Rubinstein. A Course in Game theory. MIT Press, 1994.

[11] sandvine. 2016 - global internet phenomena. https://www.sandvine.com/hubfs/ downloads/archive/2016-global-internet-phenomena-report-latin-america-and-north-america. pdf, 2016.

[12] T. Wu. Network neutrality, broadband discrimination. Journal of Telecommunications and High Technology, 2(1):141-176, 2003.

\section{A Proof of Lemma 2}

Proof: Assume that there is an equilibrium for which both ISPs offer equal qualities, for each CP, i.e., $Q_{A 1}=Q_{B 1}$ and $Q_{A 2}=Q_{B 2}$. This means that all users are indifferent between both ISPs. From the expressions of qualities (1), the two following conditions must hold:

$$
\begin{aligned}
T_{A 1} & =\frac{\beta_{A} C_{A}}{\beta_{B} C_{B}} T_{B 1} \\
T_{A 2} & =\frac{\left(1-\beta_{A}\right) C_{A}}{\left(1-\beta_{B}\right) C_{B}} T_{B 2} .
\end{aligned}
$$

Note that $\bar{\theta}=\mathbb{E}[\theta]$ is the total demand mass for CP1, i.e., the aggregated proportion of users using $\mathrm{CP} 1$ services during the peak hour. As a consequence, the demand mass for $\mathrm{CP} 2$ is $1-\bar{\theta}$.

From the repartition of users between ISPs, those demand masses should verify

$$
\begin{aligned}
\frac{T_{A 1}}{\gamma}+\frac{T_{B 1}}{\gamma} & =\bar{\theta} \\
T_{A 2}+T_{B 2} & =1-\bar{\theta} .
\end{aligned}
$$

Those equations are easy to solve:

- (16) and (18) directly give $\left\{\begin{array}{l}T_{A 1}=\alpha_{1} \gamma \bar{\theta} \\ T_{B 1}=\left(1-\alpha_{1}\right) \gamma \bar{\theta}\end{array}\right.$, that is, the first part in (9);

- Similarly, (17) and (19) yield $\left\{\begin{array}{l}T_{A 2}=\alpha_{2}(1-\bar{\theta}) \\ T_{B 2}=\left(1-\alpha_{2}\right)(1-\bar{\theta})\end{array}\right.$, i.e., the second part of (9).

From those unique solutions, one can directly compute the market share of each ISP:

$$
\left\{\begin{array}{l}
m_{A}=\frac{T_{A 1}}{\gamma}+T_{A 2}=\alpha_{1} \bar{\theta}+\alpha_{2}(1-\bar{\theta}) \\
m_{B}=\frac{T_{B 1}}{\gamma}+T_{B 2}=\left(1-\alpha_{1}\right) \bar{\theta}+\left(1-\alpha_{2}\right)(1-\bar{\theta})=1-\alpha_{1} \bar{\theta}-\alpha_{2}(1-\bar{\theta}),
\end{array}\right.
$$

yielding (10).

Note however that not all values of $T_{A 1}, T_{A 2}, T_{B 1}$, and $T_{B 2}$ can be obtained by choosing the set $\Theta_{A}$ and its complement $\Theta_{B}$. Indeed, for a given mass $m_{A}$ of ISP $A$ subscribers, we must have 
$\frac{T_{A 1}}{\gamma} \geq \int_{0}^{m_{A}} \theta \mathrm{d} F(\theta)=G\left(m_{A}\right)$, since in the right-hand side we only take the users with minimum values of $\theta$, hence minimizing $T_{A 1}$ under the constraint that a mass $m_{A}$ subscribes to ISP $A$. This implies that the actual value of $m_{A}$ satisfies

$$
m_{A} \leq G^{-1}\left(T_{A 1} / \gamma\right)
$$

where we can check that the argument of $G^{-1}$ is in $[0, \bar{\theta}]$ because $T_{A 1} / \gamma$ cannot exceed the total demand for CP1, that is $\bar{\theta}$.

Similarly, with the same mass $m_{A}$, we necessarily have $T_{A 2} \leq \int_{0}^{m_{A}}(1-\theta) \mathrm{d} F(\theta)=F\left(m_{A}\right)-$ $G\left(m_{A}\right)$ since the right-hand side assumes $\Theta_{A}$ is made of the users most interested in CP2. Noting that the integral is an increasing function of $m_{A}$, we obtain by injecting (20)

$$
T_{A 2} \leq F\left(m_{A}\right)-G\left(m_{A}\right) \leq F\left(G^{-1}\left(T_{A 1} / \gamma\right)\right)-T_{A 1} / \gamma .
$$

Noting that $m_{A}=T_{A 1} / \gamma+T_{A 2}$, we find that a necessary condition for having an equilibrium where users are indifferent between both ISPs is that

$$
m_{A} \leq F\left(G^{-1}\left(T_{A 1} / \gamma\right)\right) .
$$

Replacing the values of $m_{A}$ and $T_{A 1} / \gamma$ from (10) and (9) yields

$$
\alpha_{1} \bar{\theta}+\alpha_{2}(1-\bar{\theta}) \leq F\left(G^{-1}\left(\alpha_{1} \bar{\theta}\right)\right),
$$

that is the right-hand inequality in (8).

To get the left-hand side inequality of (8), we apply the same reasoning but upper-bounding $T_{A 1}$ and lower-bounding $T_{A 2}$. Indeed for a given $m_{A}$ we must have

$$
T_{A 1} / \gamma \leq \int_{1-m_{A}}^{1} \theta \mathrm{d} F(\theta)=\bar{\theta}-\int_{0}^{1-m_{A}} \theta \mathrm{d} F(\theta)=\bar{\theta}-G\left(1-m_{A}\right)=\bar{\theta}-G\left(m_{B}\right)
$$

where we used the fact that $m_{A}+m_{B}=1$ for the last equality. Hence

$$
m_{B} \leq G^{-1}\left(\bar{\theta}-T_{A 1} / \gamma\right)=G^{-1}\left(T_{B 1} / \gamma\right),
$$

since $T_{A 1} / \gamma+T_{B 1} / \gamma=\bar{\theta}$; again the argument of $G^{-1}$ is in $[0, \bar{\theta}]$.

On the other hand, lower-bounding $T_{A 2}$ leads to

$$
T_{A 2} \geq \int_{1-m_{A}}^{1}(1-\theta) \mathrm{d} F(\theta)=1-\bar{\theta}-\int_{0}^{1-m_{A}}(1-\theta) \mathrm{d} F(\theta)=1-\bar{\theta}-F\left(m_{B}\right)+G\left(m_{B}\right),
$$

where we used again $m_{A}+m_{B}=1$. The function $m \mapsto \int_{0}^{m}(1-\theta) \mathrm{d} F(\theta)$ being increasing, we get from $(21)$

$$
T_{A 2} \geq 1-\bar{\theta}-F\left(G^{-1}\left(T_{B 1} / \gamma\right)\right)+T_{B 1} / \gamma
$$

which we can rewrite, since $T_{A 1} / \gamma+T_{B 1} / \gamma=\bar{\theta}$, as

$$
\underbrace{T_{A 1} / \gamma+T_{A 2}}_{=m_{A}} \geq 1-F\left(G^{-1}\left(T_{B 1} / \gamma\right)\right) .
$$

Finally, taking the values of $m_{A}$ and $T_{A 1} / \gamma$ from (10) and (9) yields

$$
\alpha_{1} \bar{\theta}+\alpha_{2}(1-\bar{\theta}) \geq 1-F\left(G^{-1}\left(\left(1-\alpha_{1}\right) \bar{\theta}\right)\right),
$$

i.e., the left-hand inequality of (8).

Note that we could run the same reasoning by focusing on $\operatorname{ISP} B$, but this would lead to the exact two inequalities summarized in (8), since for example when we upper-bounded $T_{A 1}$ we were also lower-bounding $T_{B 1}$, their sum being the constant $\gamma \bar{\theta}$.

Finally, to end the proof we need to show that (8) is not only necessary, but also sufficient to have an equilibrium with all users indifferent between both ISPs. More specifically, under that condition there needs to be a partition of the interval $[0,1]$ into two sets $\Theta_{A}$ and $\Theta_{B}$ giving the values of $T_{i, j}$ expressed in (9). ${ }^{1}$

\footnotetext{
${ }^{1}$ From Patrick: La je suis sur que ca marche, mais je sais pas comment le prouver tres proprement. Revenir dessus plus tard, ou bien dire que c'est "straightforward"...?
} 


\section{B Proof of Lemma 3}

Proof: From (3), if there is an equilibrium with $Q_{A 1}>Q_{B 1}$ and $Q_{A 2}<Q_{B 2}$, then it is of the form $\left\{\begin{array}{l}\Theta_{A}=\left[\theta^{*}, 1\right] \\ \Theta_{B}=\left[0, \theta^{*}\right)\end{array}\right.$, with $\theta^{*}$ such that $\theta^{*}$-type users are indifferent between both ISPs (we affect them here to ISP $A$, without any impact since their mass is 0 under Assumption A). Indeed, a type- $\theta$ user prefers $A$ over $B$ if

$$
\begin{aligned}
\theta Q_{A 1}+(1-\theta) Q_{A 2} & \geq \theta Q_{B 1}+(1-\theta) Q_{B 2} \\
\Leftrightarrow \quad \theta & \geq \frac{Q_{B 2}-Q_{A 2}}{Q_{A 1}-Q_{B 1}+Q_{B 2}-Q_{A 1}}:=\theta^{*} .
\end{aligned}
$$

Let us now compute the different traffic loads as functions of $\theta^{*}$ :

$$
\left\{\begin{array}{l}
T_{A 1}=\gamma \int_{\theta^{*}}^{1} \theta \mathrm{d} F(\theta)=\gamma\left(\bar{\theta}-G\left(\theta^{*}\right)\right) \\
T_{A 2}=\int_{\theta^{*}}^{1}(1-\theta) \mathrm{d} F(\theta)=1-\bar{\theta}-\left(F\left(\theta^{*}\right)-G\left(\theta^{*}\right)\right) \\
T_{B 1}=\gamma \int_{0}^{\theta^{*}} \theta \mathrm{d} F(\theta)=\gamma G\left(\theta^{*}\right) \\
T_{B 2}=\int_{0}^{\theta^{*}}(1-\theta) \mathrm{d} F(\theta)=F\left(\theta^{*}\right)-G\left(\theta^{*}\right) .
\end{array}\right.
$$

Using the functions $G$ and $H$ from Definition 4 (from which we also get $G(1)=\bar{\theta}$ and $H(1)=1-\bar{\theta}$ ), those relations can be written as follows:

$$
\left\{\begin{aligned}
T_{A 1} / \gamma & =G(1)-G\left(\theta^{*}\right) \\
T_{A 2} & =H(1)-H\left(\theta^{*}\right) \\
T_{B 1} / \gamma & =G\left(\theta^{*}\right) \\
T_{B 2} & =H\left(\theta^{*}\right) .
\end{aligned}\right.
$$

From (1), we get $Q_{A 1}=\frac{\beta_{A} C_{A}}{\gamma\left(G(1)-G\left(\theta^{*}\right)\right)}, Q_{B 1}=\frac{\beta_{B} C_{B}}{\gamma G\left(\theta^{*}\right)}, Q_{A 2}=\frac{\left(1-\beta_{A}\right) C_{A}}{H(1)-H\left(\theta^{*}\right)}, Q_{B 2}=\frac{\left(1-\beta_{B}\right) C_{B}}{H\left(\theta^{*}\right)}$. Plugging it into (22), $\theta^{*}$ should be a solution of the equation

$$
x=\frac{1}{1+\frac{1}{\gamma} \frac{\frac{\beta_{A} C_{A}}{G(1)-G(x)}-\frac{\beta_{B} C_{B}}{G(x)}}{\frac{\left(1-\beta_{B}\right) C_{B}}{H(x)}-\frac{\left(1-\beta_{A}\right) C_{A}}{H(1)-H(x)}}}
$$

such that the inequalities on qualities are satisfied, i.e., such that $\frac{\beta_{A} C_{A}}{G(1)-G\left(\theta^{*}\right)}>\frac{\beta_{B} C_{B}}{G\left(\theta^{*}\right)}$ and $\frac{\left(1-\beta_{B}\right) C_{B}}{H\left(\theta^{*}\right)}>\frac{\left(1-\beta_{A}\right) C_{A}}{H(1)-H\left(\theta^{*}\right)}$.

The first of those inequalities is equivalent to (using $G(1)=\bar{\theta}$ and the definition of $\alpha_{1}$ )

$$
G\left(\theta^{*}\right)>\left(1-\alpha_{1}\right) \bar{\theta}
$$

Similarly, the second inequality is equivalent to

$$
H\left(\theta^{*}\right)<\left(1-\alpha_{2}\right)(1-\bar{\theta}),
$$

so we can summarize the two conditions into

$$
G^{-1}\left(\left(1-\alpha_{1}\right) \bar{\theta}\right)<\theta^{*}<H^{-1}\left(\left(1-\alpha_{2}\right)(1-\bar{\theta})\right) .
$$

Therefore, an equilibrium of the type we are looking for can only exist if $G^{-1}\left(\left(1-\alpha_{1}\right) \bar{\theta}\right)<$ $\left.H^{-1}\left(\left(1-\alpha_{2}\right)(1-\bar{\theta})\right)\right)$.

If it is the case, then on the open interval $\left(G^{-1}\left(\left(1-\alpha_{1}\right) \bar{\theta}\right), H^{-1}\left(\left(1-\alpha_{2}\right)(1-\bar{\theta})\right)\right)$ the righthand side of (25) is continuous and strictly decreasing with limit values 1 and 0 , hence (25) has a unique solution, which we can denote by $\theta^{*}$ and gives the equilibrium sets $\Theta_{A}=\left[\theta^{*}, 1\right]$ and $\Theta_{B}=\left[0, \theta^{*}\right)$. 
Finally, we notice that we can rewrite the condition for such an equilibrium to exist:

$$
\begin{aligned}
& G^{-1}\left(\left(1-\alpha_{1}\right) \bar{\theta}\right) & <H^{-1}\left(\left(1-\alpha_{2}\right)(1-\bar{\theta})\right) \\
& \Leftrightarrow F\left(G^{-1}\left(\left(1-\alpha_{1}\right) \bar{\theta}\right)\right)-\left(1-\alpha_{1}\right) \bar{\theta} & <\left(1-\alpha_{2}\right)(1-\bar{\theta}) \\
\Leftrightarrow & F\left(G^{-1}\left(\left(1-\alpha_{1}\right) \bar{\theta}\right)\right) & <1-\left(\alpha_{1} \bar{\theta}+\alpha_{2}(1-\bar{\theta})\right),
\end{aligned}
$$

where we used the facts that $H$ is an increasing bijection, and that $H(x)=F(x)-G(x)$. 